\title{
Diamond-Blackfan anemia caused by chromosome 1p22 deletion encompassing RPL5
}

\author{
Makiko Tominaga ${ }^{1,2}$, Satoshi Hamanoue ${ }^{3}$, Hiroaki Goto ${ }^{3}$, Toshiyuki Saito ${ }^{4}$, Jun-ichi Nagai ${ }^{4}$, Mitsuo Masuno ${ }^{5}$,
} You Umeda ${ }^{2}$ and Kenji Kurosawa (D)

\begin{abstract}
Diamond-Blackfan anemia (DBA) is an inherited anemia with multiple congenital malformations, and mutations in ribosomal protein genes have been identified as the underlying cause. We describe a female patient with mild DBA due to 1 p22 deletion, encompassing the gene encoding 60S ribosomal protein L5 (RPL5). Considering previously reported cases together with our patient, we suggest that RPL5 haploinsufficiency might cause a less severe form of DBA than loss-of-function mutations.
\end{abstract}

Diamond-Blackfan anemia (DBA) [MIM. 105650] is an autosomal dominant disorder characterized by severe normochromic and macrocytic anemia with normal leukocytes and platelets, congenital malformations, and growth retardation. The phenotype varies from mild to severe fetal anemia ${ }^{1}$, and DBA is associated with an increased risk of hematological malignancy ${ }^{1}$. Mutations in 19 genes encoding ribosomal proteins have been recognized as causing $\mathrm{DBA}^{2}$. The mutations reported to date include single-nucleotide variants and copy-number variants, both of which result in loss-of-function or haploinsufficiency of the causal genes ${ }^{2-6}$. Although mutations in $R P L 5$, encoding $60 \mathrm{~S}$ ribosomal protein L5, account for $11 \%$ of the patients with $\mathrm{DBA}^{2,7}$, only three patients have been reported to have a large deletion of $R P L 5^{4,6,8,9}$. Here, we report a female patient with DBA caused by $1 \mathrm{p} 22$ deletion, and we attempt to elucidate the clinical and hematological features of this large deletion encompassing RPL5.

\footnotetext{
Correspondence: Kenji Kurosawa (kkurosawa@kcmc.jp)

'Division of Medical Genetics, Kanagawa Children's Medical Center, Yokohama, Japan

${ }^{2}$ Children's Medical Center, Showa University Northern Yokohama Hospital, Yokohama, Japan
}

Full list of author information is available at the end of the article.
The proposita was a 20-year-old woman. She was born at 39-weeks gestation after an uneventful pregnancy. At birth, her weight was $2055 \mathrm{~g}(-2.3 \mathrm{SD})$, and her length was $48 \mathrm{~cm}(-0.2 \mathrm{SD})$; her occipitofrontal circumference (OFC) was $32 \mathrm{~cm}(-0.6 \mathrm{SD})$. She underwent ligation of the patent ductus arteriosus on day 27; total repair of her atrial septal defect occurred at 1 year 9 months. At her first visit to our genetics clinic at 3 years of age, her weight and height were $11.1 \mathrm{~kg}(-1.2 \mathrm{SD})$ and $84.4 \mathrm{~cm}(-2.3 \mathrm{SD})$, respectively, with an OFC of $52.4 \mathrm{~cm}(+2.5 \mathrm{SD})$. Her facial appearance characteristics included typical downslanting palpebral fissures, deep-set eyes, a thin upper lip, and macrocephaly. Hypoplastic finger-like thumbs with nail hypoplasia were noted (Fig. 1a). Brain magnetic resonance imaging revealed cortical atrophy and dilated ventricles. Her developmental milestones were delayed, with head control at 9 months, rolling over at 1 year, and walking without support at 4 years. At the age of 6 years, she was noted as having mild anemia: hemoglobin $(\mathrm{Hb})$, $10.5 \mathrm{~g} / \mathrm{dL}$; mean corpuscular volume (MCV), $89 \mathrm{fL}$; mean corpuscular hemoglobin $(\mathrm{MCH}), 30.2 \mathrm{pg}$; white blood cell (WBC) count, 5500/cumm; and platelet count, $32.2 \times 10^{4}$. At the age of 16 years, her anemia continued, with values as follows: $\mathrm{Hb}, 8.8 \mathrm{~g} / \mathrm{dL}$; MCV, $92 \mathrm{fL} ; \mathrm{MCH}, 31.8 \mathrm{pg}$; WBC count, 3200/cumm (lymphocytes $48 \%$, neutrophils $41 \%$ );

\section{(c) The Author(s) 2019}

(c) Open Access This article is licensed under a Creative Commons Attribution 4.0 International License, which permits use, sharing, adaptation, distribution and reproduction cc) in any medium or format, as long as you give appropriate credit to the original author(s) and the source, provide a link to the Creative Commons license, and indicate if changes were made. The images or other third party material in this article are included in the article's Creative Commons license, unless indicated otherwise in a credit line to the material. If material is not included in the article's Creative Commons license and your intended use is not permitted by statutory regulation or exceeds the permitted use, you will need to obtain permission directly from the copyright holder. To view a copy of this license, visit http://creativecommons.org/licenses/by/4.0/. 
a

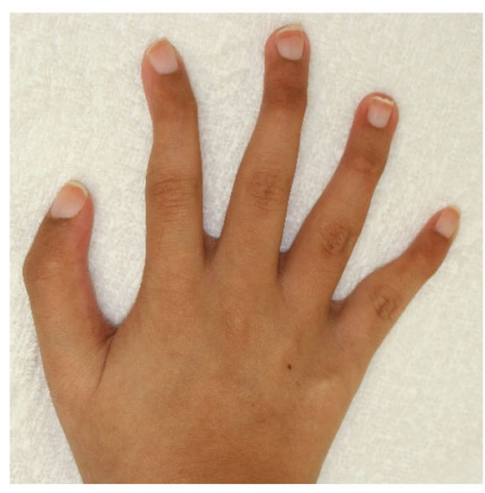

d

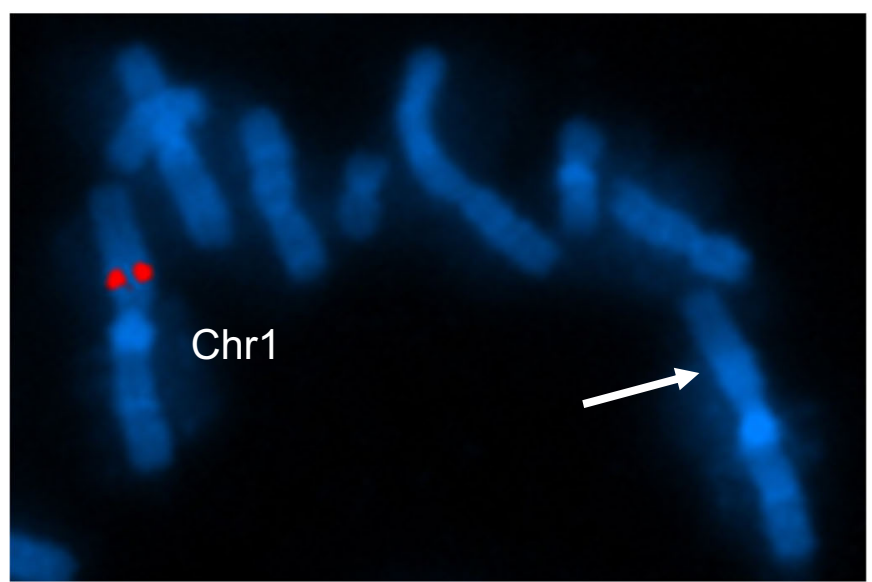

b

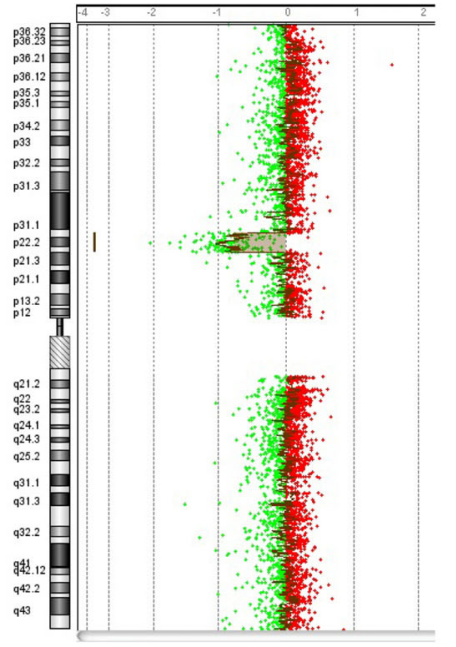

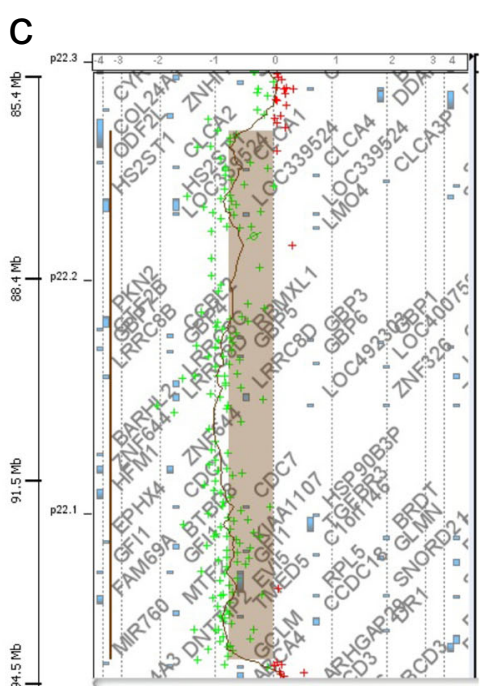

e

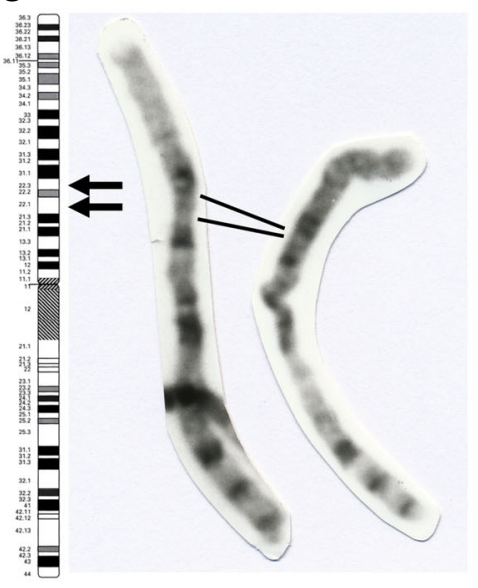

Fig. 1 Clinical phenotype and molecular cytogenetic findings. a Hypoplastic thumb (triphalangeal thumb) was noted. b, c Array CGH analysis showing the 7.9-Mb deleted region at 1p22.1-p22.3. d Partial image of metaphase fluorescence in situ hybridization (FISH) of lymphocytes using the RP11-62M16 BAC clone (chr1: 92381303-92517650, NCBI35/hg17) as a specific probe for 1p22.1 (red). One signal was observed for the patient, consistent with a deletion at 1p22.1-p22.3. The signal of RP11-62M16 was absent from derivative chromosome 1 (arrow). e Retrospective evaluation of G-banded metaphase chromosome 1 revealed a heterozygous deletion of 1p22.1-p22.3 (arrows)

and platelet count, $32.4 \times 10^{4}$. Her bone marrow had a markedly hypocellular appearance, with a small number of erythroid and myeloid cells and megakaryocytes. Normal values in cytogenetic stress testing were obtained for mitomycin C, bleomycin, cyclophosphamide, diepoxybutane, and fludarabine. She also had primary amenorrhea and underwent vaginal fenestration for hematometrocolpos due to vaginal atresia at 16 years. At age 20, the following were recorded:Hb, $9.5 \mathrm{~g} / \mathrm{dL} ; \mathrm{MCV}$, $92.7 \mathrm{fL} ; \mathrm{MCH}, 30.4 \mathrm{pg}$; WBC count, 3100/cumm; and platelet count, $29.1 \times 10^{4}$. To date, she has not required transfusion for her mild hypochromic anemia.

Written informed consent was obtained from the parents of the patient, and this study was performed in accordance with the Kanagawa Children's Medical Center Review Board and Ethics Committee. Array comparative genomic hybridization (array CGH) using Agilent SurePrint G3 Human CGH Microarray Kit $8 \times 60 \mathrm{~K}$ (Agilent Technologies, Inc., Santa Clara, CA, USA) revealed a 7.9-Mb deletion (arr[GRCh37] 1p22.3p22.1(86369841_ 94276387)x1) (Fig. 1b, c) ${ }^{10}$. No other genomic imbalances were identified based on the array analysis. Fluorescence in situ hybridization (FISH) analysis with relevant bacterial artificial chromosome (BAC) clones confirmed the deletion (Fig. 1d). Both parents refused cytogenetic evaluation. Further retrospective evaluation of G-banded metaphase chromosome 1 revealed a heterozygous deletion of 1p22.1-p22.3 (Fig. 1e). 


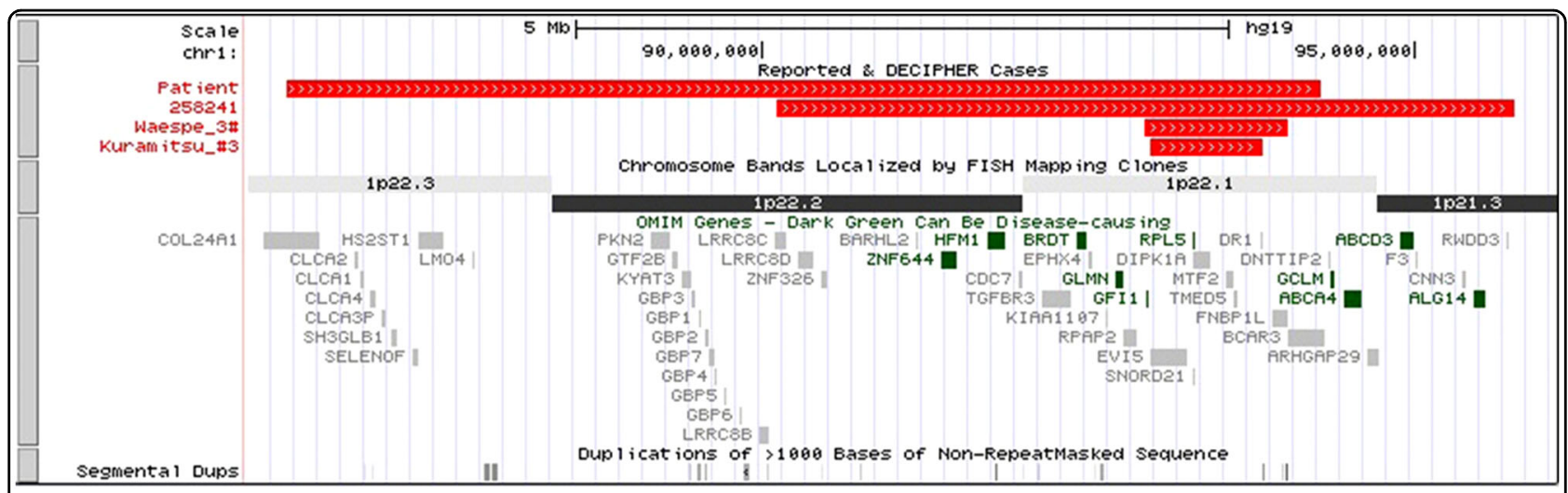

Fig. 2 Schematic representation of the 1p21.3-p22.3 deletions in the present case, DECIPHER patient (2258241), and previously reported cases encompassing RPL5 based on USCS Genome Browser 2009 (GRCh37/hg19) Assembly (http://www.genome.ucsc.edu)

The patient exhibited variable clinical manifestations, such as multiple congenital anomalies, moderate to severe developmental delay, and characteristic hematological findings of mild normochromic anemia and neutropenia. The clinical features overlapped with those of DBA and Fanconi syndrome. However, hematological analysis excluded the possibility of Fanconi anemia. The array CGH analysis revealed a 7.9-Mb deletion of 1p22.1-p22.3 encompassing 40 OMIM genes, including RPL5. To our knowledge, only three cases with large deletions of RPL5 associated with DBA have been reported ${ }^{4,6,8,9}$. In general, most patients with DBA show a steroid-dependent or transfusion-dependent clinical course. Although the detailed clinical and hematological features of the three patients with RPL5 haploinsufficiency are not available, two patients were reported to be steroid responsive ${ }^{4,8}$. Considering those cases together with our patient who showed a mild form of DBA without hematological treatments, we believe that RPL5 haploinsufficiency might result in a less severe form of DBA than that caused by loss-of-function mutations. Although the involvement of neighboring genes could not be proven in the etiology of the patient's phenotype, our case provides crucial information on the underlying mechanism for DBA (Fig. 2). Further information on DBA associated with genetic studies is required for a clearer understanding of the genetic and molecular bases of DBA.

\section{HGV database}

The relevant data from this Data Report are hosted at the Human Genome Variation Database at https://doi.org/10.6084/m9.figshare.hgv.2594.

\section{Acknowledgements}

We greatly appreciate the family members of the patient for allowing us to publish this report. This research was supported by Research on Rare and Intractable Diseases from the Ministry of Health, Labor and Welfare, Japan, Japan Agency for Medical Research and Development (AMED) grant number 18kk0205014; JSPS KAKENHI 17K10069 (K.K.).

\section{Author details}

'Division of Medical Genetics, Kanagawa Children's Medical Center, Yokohama, Japan. ${ }^{2}$ Children's Medical Center, Showa University Northern Yokohama Hospital, Yokohama, Japan. ${ }^{3}$ Division of Hematology and Oncology, Kanagawa Children's Medical Center, Yokohama, Japan. ${ }^{4}$ Department of Clinical Laboratory, Kanagawa Children's Medical Center, Yokohama, Japan. ${ }^{5}$ Genetic Counseling Program, Kawasaki University of Medical Welfare, Kurashiki, Japan

\section{Conflict of interest}

The authors declare that they have no conflict of interest.

\section{Publisher's note}

Springer Nature remains neutral with regard to jurisdictional claims in published maps and institutional affiliations.

Received: 15 June 2019 Revised: 5 July 2019 Accepted: 7 July 2019. Published online: 8 August 2019

\section{References}

1. Ball, S. Diamond Blackfan anemia. Hematol. Am. Soc. Hematol. Educ. Program 2011, 487-491 (2011).

2. Ulirsch, J. C. et al. The genetic landscape of Diamond-Blackfan anemia. Am. J. Hum. Genet. 103, 930-947 (2018).

3. Boria, I. et al. The ribosomal basis of Diamond-Blackfan Anemia: mutation and database update. Hum. Mutat. 31, 1269-1279 (2010).

4. Kuramitsu, M. et al. Extensive gene deletions in Japanese patients with Diamond-Blackfan anemia. Blood 119, 2376-2384 (2012).

5. Farrar, J. E. et al. Ribosomal protein gene deletions in Diamond-Blackfan anemia. Blood 118, 6943-6951 (2011).

6. Arbiv, O. A. et al. Molecular analysis and genotype-phenotype correlation of Diamond-Blackfan anemia. Clin. Genet. 93, 320-328 (2018).

7. Gazda, H. T. et al. Ribosomal protein $L 5$ and $L 11$ mutations are associated with cleft palate and abnormal thumbs in Diamond-Blackfan anemia patients. Am. J. Hum. Genet. 83, 769-780 (2008).

8. Quarello, P. et al. High frequency of ribosomal protein gene deletions in Italian Diamond-Blackfan anemia patients detected by multiplex ligation-dependent probe amplification assay. Haematologica 97, 1813-1817 (2012)

9. Waespe, N. et al. The clinical impact of copy number variants in inherited bone marrow failure syndromes. NPJ Genom. Med. 2, 18 (2017).

10. Ono, H., Kurosawa, K., Wakamatsu, N. \& Masuda, S. Hearing impairment in a female infant with interstitial deletion of 2q24.1q24.3. Congenit. Anom. (Kyoto) 57, 118-121 (2017). 